\title{
Correction to: Reassessment of the 1907 Sumatra "Tsunami Earthquake" Based on Macroseismic, Seismological, and Tsunami Observations, and Modeling
}

\author{
Stacey Servito Martin, ${ }^{1}$ (i) Linlin Li, ${ }^{1}$ Emile A. Okal, ${ }^{2}$ Julie Morin, ${ }^{3}$ Alexander E. G. Tetteroo, ${ }^{4}$ \\ Adam D. Switzer, ${ }^{1,5}$ and Kerry E. Sieh ${ }^{1,5}$
}

Correction to: Pure Appl. Geophys. 176 (2019),

$2831-2868$

https://doi.org/10.1007/s00024-019-02134-2

Due to an unfortunate oversight we did not publish the hypothetical slip model we used in Figure 9a in our original article on the 1907 Sumatra tsunami earthquake in Indonesia. We acknowledge this omission on our part and include it here in the Generic Mapping Tools (GMT) format with slip values $(-\mathrm{Z})$ given in meters.

The original article can be found online at https://doi.org/10.1007/ s00024-019-02134-2.

1 Earth Observatory of Singapore, Nanyang Technological University, Singapore, Singapore. E-mail: 7point1@gmail.com; smartin@ntu.edu.sg

2 Department of Earth and Planetary Sciences, Northwestern University, Evanston, USA.

3 Laboratoire Magmas et Volcans, Université Clermont Auvergne, Clermont-Ferrand, France.

4 Institute for History, Leiden University, Leiden, The Netherlands.

5 Asian School of the Environment, Nanyang Technological University, Singapore, Singapore. 


$$
\begin{aligned}
& >- \text { Z } 15.000000 \\
& 96.737553 \quad 0.730344 \\
& 96.6336720 .966045 \\
& 96.9346591 .098649 \\
& 97.0385210 .862948 \\
& >- \text { Z } 0.100000 \\
& 96.969748 \quad 0.964243 \\
& 96.8658561 .199945 \\
& 97.166867 \quad 1.332548 \\
& 97.270734 \quad 1.096847 \\
& >-\mathrm{Z} 15.000000 \\
& 96.648214 \quad 0.941171 \\
& 96.4855031 .180619 \\
& 96.7575991 .365420 \\
& 96.920288 \quad 1.125972 \\
& >-\mathrm{Z} 0.100000 \\
& 96.880414 \quad 1.175072 \\
& 96.7176851 .414520 \\
& 96.989803 \quad 1.599317 \\
& 97.152504 \quad 1.359869 \\
& >- \text { Z } 9.000000 \\
& 96.495834 \quad 1.168151 \\
& 96.3278921 .367236 \\
& 96.5793811 .579239 \\
& 96.7473011 .380154 \\
& >-\mathrm{Z} 0.100000 \\
& 96.7280321 .402052 \\
& 96.5600711 .601137 \\
& 96.8115851 .813137 \\
& 96.9795211 .614052 \\
& >- \text { Z } 9.000000 \\
& 96.3449691 .350820 \\
& 96.1206691 .548484 \\
& 96.3382441 .795169 \\
& 96.5625231 .597505 \\
& >-\mathrm{Z} 0.100000 \\
& 96.577171 \quad 1.584719 \\
& 96.3528421 .782383 \\
& 96.570444 \quad 2.029067 \\
& 96.7947491 .831403 \\
& >- \text { Z } 9.000000 \\
& 96.126285 \quad 1.544575 \\
& 95.9123551 .717045 \\
& 96.1189291 .973013 \\
& 96.332840 \quad 1.800544 \\
& >-\mathrm{Z} 0.100000 \\
& 96.358387 \quad 1.778575 \\
& 96.144427 \quad 1.951045 \\
& 96.3510292 .207011 \\
& 96.5649672 .034542
\end{aligned}
$$

$$
\begin{aligned}
& >-Z 24.000000 \\
& 95.9004561 .728285 \\
& 95.699801 \quad 1.924861 \\
& 95.9301612 .159701 \\
& 96.1307891 .963125 \\
& >-\mathrm{Z} 0.100000 \\
& 96.132555 \quad 1.962284 \\
& 95.9318692 .158860 \\
& 96.162265 \quad 2.393700 \\
& 96.3629212 .197125 \\
& >- \text { Z } 24.000000 \\
& 95.714071 \quad 1.913068 \\
& 95.503097 \quad 2.076772 \\
& 95.704945 \quad 2.336519 \\
& 95.915898 \quad 2.172814 \\
& >- \text { Z } 0.100000 \\
& 95.946173 \quad 2.147067 \\
& 95.735164 \quad 2.310771 \\
& 95.937045 \quad 2.570518 \\
& 96.1480312 .406813 \\
& >- \text { Z } 3.000000 \\
& 95.549002 \quad 2.051863 \\
& 95.2421592 .160858 \\
& 95.352403 \quad 2.470718 \\
& 95.659237 \quad 2.361724 \\
& >- \text { Z } 0.100000 \\
& 95.781018 \quad 2.285962 \\
& 95.474123 \quad 2.394956 \\
& 95.584387 \quad 2.704818 \\
& 95.891273 \quad 2.595824
\end{aligned}
$$

Publisher's Note Springer Nature remains neutral with regard to jurisdictional claims in published maps and institutional affiliations. 